\title{
STRAF- UND BUBGELDRECHTLICHE VERANTWORTUNG VON VERBÄNDEN IM GELDWÄSCHESTRAFRECHT
}

\author{
Criminal and administrative responsibility of companies \\ in money laundering criminal law
}

\author{
JUDIT JACSÓ*
}

\begin{abstract}
There are several points of contact between money laundering and compliance. There are risks associated with non-compliance in the area of money laundering criminal law. For example, the liability of the management or employees of the company or the company itself under criminal law and legal fines. The first part of the article deals with conceptual clarification and the roots of money laundering and compliance. The second part analysed the fight against money laundering, including money laundering compliance. After the introductory chapters, an important aim of the article is to examine the responsibility of companies for compliance with money laundering regulations, which is strongly influenced by the requirements of the European Union.
\end{abstract}

Keywords: money laundering, compliance, criminal compliance, liability of company, administrative responsibility

\section{EINLEITUNG}

Nimmt man den Werdegang des Deliktes der Geldwäsche und deren Bekämpfung unter die Lupe, bemerkt man, dass kaum ein anderes Delikt so schnell in den Mittelpunkt internationaler und nationaler Bemühungen getreten ist und heute noch im Mittelpunkt diverser Bestrebungen steht. Der Gesetzgeber der Europäischen Union hat ebenso früh erkannt, dass es erforderlich ist, parallel zum wirtschaftlichen Integrationsprozess innerhalb der Europäischen Union auch ein entsprechendes Abwehrsystem aufzubauen, das verhindert, dass die Straftäter von den gemeinschaftsrechtlichen Grundfreiheiten profitieren können. Die Harmonisierung der mitgliedstaatlichen Geldwäsche-Regelungen hat in Europa in den 90-er Jahren begonnen. Das nationale Geldwäschestrafrecht ist unter starkem supranationalen und internationalen Einfluss ${ }^{1}$ eingeführt worden.

* Universitätsprofessorin, Lehrstuhlleiterin, Universität Miskolc, Staats- und Rechtswissenschaftliche Fakultät, Institute für Strafrechtswissenschaften, Lehrstuhl für Strafrecht und Kriminologie.

1 Hervorzuheben sind hier die repressiven Vorgaben der Vereinten Nationen (UNO) im Wiener Übereinkommen von 1988, wodurch zum ersten Mal gegen der mit Drogengeldern verbundenen Geldwäsche durch genaue Formulierung des Geldwäschetatbestandes entgegengetreten wurde. Im Palermo Übereinkommen aus dem Jahre 2000 wurde die 
Es gibt mehrere Berührungspunkte zwischen Geldwäsche und Compliance. Compliance ist in Europa heute schon in den täglichen Sprachgebrauch übernommen worden. Besonders in bestimmten Branchen (bei Akteuren des Finanz- und Wirtschaftslebens) stieg seine Bedeutung in der letzten Zeit. Compliance und die damit verbundenen Fragen erweckten auch das Interesse der Wissenschaft. ${ }^{2}$ Compliance als Begriff stammt aus dem anglo-amerikanischen Rechtssystem. Mit dem NonCompliance-Verhalten im Bereich des Geldwäschestrafrechts sind Risiken verbunden, wie die strafrechtliche und bußgeldrechtliche Haftung der Leitung oder Mitarbeiter des Unternehmens oder des Unternehmens selbst. Der erste Teil des Beitrages befasst sich mit begrifflicher Klarstellung und dem Ursprung der Geldwäsche bzw. der Compliance. Im zweiten Teil geht es um die Geldwäschebekämpfung ${ }^{3}$, wobei sich auf die Geldwäsche Compliance fokussiert und ihre Bedeutung erläutert wird. Wichtiges Ziel des Beitrages ist es, nach den einführenden Kapiteln die Verantwortung von Unternehmen für die Einhaltung der Geldwäschevorschriften, welche durch Vorgaben der Europäischen Union stark geprägt sind, zu untersuchen.

\section{BEGRIFF UND WURZEL DER GELDWÄSCHE UND DER GELDWÄSCHEBE- KÄMPFUNG}

Der Begriff der Geldwäsche hat in den vergangenen Jahrzehnten Eingang in unseren Sprachgebrauch gefunden. Er wird in internationalen und europäischen Vorgaben bestimmt. Daneben stellt die Geldwäsche eine Straftat dar, die sowohl zum Katalog der sog. „Unionsdelikte“ zählt ${ }^{4}$, als auch in den nationalen Strafgesetzbüchern der

Geldwäsche als eine der Schlüsseldeliktskategorien der Organisierten Kriminalität festgelegt. Der Europarat hat sich ebenfalls mit der Problematik der Geldwäsche befasst (Straßburger Übereinkommen von 1990, Warschauer Übereinkommen). Als Triebfeder der Geldwäschebekämpfung dienen die Vierzig Empfehlungen der Finanziellen Arbeitsgruppe zur Bekämpfung der Geldwäsche (FATF).

2 In Ungarn sind in den letzten Jahren zwei Fachbücher und wissenschaftliche Abhandlungen darüber veröffentlich worden. (Wie z. B. JACSÓ Judit: A compliance fogalmáról és szerepéről a gazdasági életben. Miskolci Jogi Szemle, 2019/1, Sonderausgabe, 82-91.; AMBRUS István - FARKAS Ádám: A compliance alapkérdései Az etikus vállalati müködés elmélete és gyakorlata. Wolters Kluwer Hungary Kft., Budapest, 2019; KoczISZKY, György - KARDKOVÁCS, Kolos: A compliance szerepe a közösségi értékek és érdekek védelmében. Akadémiai Kiadó, Budapest, 2020; MoLNÁR Erzsébet: Compliance és az üzleti titok. Miskolci Jogi Szemle, 2021/1, Band 2, 48-63.

3 Anti Money Laundering.

4 Seit dem Vertrag von Lissabon bestimmt Art. 83 Abs. 1 Uabs. 1 und Uabs. 2 AEUV, dass durch Richtlinien Mindestvorschriften zur Festlegung von Straftaten und Strafen in Bereichen besonders schwerer Kriminalität mit grenzüberschreitender Dimension festgelegt werden können. Art 83 AEUV ist von besonderer Bedeutung, da er die Geldwäsche explizit in die Liste der schweren Kriminalitätsbereiche aufnimmt. Die neue Kompetenzgrundlage lässt den Erlass von Richtlinien über Mindestvorschriften für Straftaten bzw. Strafen in Fällen nach der Art der schützenswerten Interessen der Europäischen Union 
Mitgliedstaaten ${ }^{5}$ geregelt wird. Die Straftatbestände sind weitgehend durch europäische Rechtsakte harmonisiert.

Die Wurzel der Geldwäschebekämpfung sowie deren Begriff sind in den USA zu finden. Die USA haben auch den Titel „Vorreiter“ im Kampf gegen die Geldwäsche verdient. ${ }^{6}$ In den 70 -er Jahren wurde die Bedeutung der Finanzwelt bei der Bekämpfung der Geldwäsche erkannt, ein Pflichtensystem der Banken zur Erfassung der verdächtigen Finanztransaktionen wurde eingeführt. 1986 wurde die Geldwäsche in den USA kriminalisiert (Money Laundering Act). ${ }^{7}$

Unter Geldwäsche versteht man aus kriminologischer Sicht kurz zusammengefasst die Verschleierung der Herkunft von Geldern, die aus kriminellen Handlungen stammen. Das Wort „Geldwäsche“ deutet darauf hin, dass es hier um „schmutziges“ Vermögen geht, welches durch verschiedene Vorgänge (wirtschaftliche oder finanzielle Transaktionen) gereinigt werden soll. Es handelt sich bei der Geldwäsche um illegales aus strafrechtswidrigem Verhalten stammendes Vermögen. Es sind darunter Handlungen zu verstehen, die nicht nur darauf abzielen, die Herkunft der illegalen Vermögenswerte zu verheimlichen, sondern sie letztendlich als aus legalen Tätigkeiten stammende Vermögenswerte erscheinen zu lassen. Dies erfolgt durch die Einschleusung von deliktisch erworbenen Vermögenswerten in den legalen Finanz- und Wirtschaftskreislauf. Bei diesem Punkt spielen die im Unternehmen eingeführten Compliance-Maßnahmen eine wichtige Rolle. Die erste Phase der Geldwäsche ist nämlich die Platzierung. Durch diesen ersten Schritt wird versucht, das schmutzige Geld durch Akteure des Finanz- und Wirtschaftslebens in den legalen Finanzkreislauf einzubringen. ${ }^{8}$

Die Geldwäsche verfügt über einen eigenständigen Charakter, durch den sie von den anderen Straftaten abweicht. Im Gegensatz zu vielen anderen kriminellen Handlungen besteht die Geldwäsche aus mehreren, vordergründig legalen Handlungsschritten (z. B. Eröffnung eines Bankkontos oder Durchführung von Überweisungen). Der Ursprung der Gelder ist jedoch durch Straftatbegehungen bemakelt. Das

zu. Siehe dazu: JACSÓ, Judit: Freiheit und Sicherheit im Spiegel der Geldwäschebekämpfung in Europa. In: KARSAI, K. - NAGY, F. - SzOMORA, Zs. (Hrsg.): Freiheit - Sicherheit(Straf)Recht Beiträge eines Humboldt-Kollegs. V\&R Unipress, Universitätsverlag Osnabrück, 2011, 103-126.

5 So z.B. im ungarischen Strafgesetzbuch (uStGB) in den $\S \S 399-400$; im deutschen Strafgesetzbuch (dStGB) in $\S 261$.

6 JACSÓ, Judit: Bekämpfung der Geldwäscherei in Europa, unter besonderer Berücksichtigung des Geldwäschestrafrechts von Österreich, der Schweiz und Ungarn (Schriftenreihe Sanktionenrecht in Europa, 5. Band, Hrsg.: DANNECKER, G. - HÖPFEL, F. - SCHWARZENEGGER, CH.). Neuer Wissenschaftlicher Verlag, Berlin-Wien-Zürich, 2007, 29.

7 ARZT, Gunther: Das schweizerische Geldwäscheverbot im Lichte der amerikanischen Erfahrungen. Schweizerische Zeitschrift für Strafrecht (ZStrR), 1996, 89-109.; GURULÉ, Jimmy: The money laundering contor act of 1986. Creating a new federal offence or merely affording federal prosecutor an alternative means of punishing specified unlawful activity? American Criminal Law Review, Vol. 32/1994-1995, 823-854.

8 Siehe zu der sog. Drei-Phasen Modelle: JACSÓ (2007): a.a.O., 33. 
strafbare Verhalten an sich stellt oft eine äußerlich sozialadäquate Handlung dar und niemand wird dadurch direkt geschädigt. Die Mithilfe von Banken und anderen Akteuren des Wirtschaftslebens ist bei der Bekämpfung dieses Phänomens unverzichtbar. Deswegen verlangt die Bekämpfung der Geldwäsche erhöhte Beachtung und ihre Regelung setzt besonderes Präventionsdenken voraus.

Es gibt noch eine wichtige Frage bezüglich der strafrechtlichen Regelung der Geldwäsche. Was die Vortaten der Geldwäsche betrifft, ist zu bemerken, dass deren wesentliche Erweiterung in den vergangenen Jahrzehnten zu beobachten war. Am Anfang waren diese noch auf die Drogenkriminalität begrenzt und heute hat sich, vorangetrieben durch internationale Bestrebungen (besonders durch $\mathrm{FATF}^{9}$ ), vordergründig der sog. ,,All-Crime-Ansatz “ durchgesetzt. Dies bedeutet, dass die bemakelten Gegenstände (Vermögenswerte), die aus irgendeiner strafbaren Handlung herrühren, taugliche Tatobjekte der Geldwäsche sein können. ${ }^{10}$ Diese systematische Änderung bringt auch erhebliche Auswirkungen auf die Anforderungen in der Geldwäscheprävention mit, sie erhöht nämlich das Risiko der Strafbarkeit wegen Geldwäsche und der bußgeldrechtlichen Verantwortung der in deren Bekämpfung einbezogenen Akteuren. In Deutschland gilt dieser Ansatz seit Inkrafttreten des Gesetzes zur Verbesserung der strafrechtlichen Bekämpfung der Geldwäsche vom 09. 03. 2021 (BGB1 I S. 327) am 18. März 2021. In Ungarn wurde der Kreis der Vortaten der Geldwäsche mit dem Gesetz Nr. CXX aus dem Jahre 1999 so geändert, dass alle mit Freiheitsstrafe zu ahndenden Delikte darunterfallen. ${ }^{11}$ Praktisch bedeutete dies die Einführung des All-Crime-Ansatzes seit dem 1. März 2000. Mit dem neuen Strafgesetzbuch aus dem Jahre 2012 wurde dann das Erfordernis der Freiheitsstrafe auch gestrichen. ${ }^{12}$ Trotzdem entdeckte Moneyval ${ }^{13}$ bei der Überprüfung der Wirksamkeit der Systeme zur Bekämpfung von Geldwäsche bei der letzten Evaluation von Ungarn Mängel, die daraufhin aber gebessert wurden (dazu gehört die konzeptionelle Neugestaltung des Straftatbestands der Geldwäsche im Jahr 2020).

9 https://www.fatf-gafi.org/ (abgerufen am 8. 7. 2021).

10 Siehe dazu kritisch BÜLTE, Jens: Reform des $§ 261$ StGB: Vermeintlich effektive Abschöpfung statt Rechtsstaatlichkeit. Geldwäsche \& Recht, 2021/1, 8.

11 Siehe zu der ungarischen Regelung der Geldwäsche: JACSÓ, Judit - UDVARHELYI, Bence: The fight against money laundering in Hungary. In: FARKAS Ákos - DANNECKER, Gerhard - JACSÓ Judit (Hrsg.): Criminal law aspects of the protection of the financial interests of the EU: with particular emphasis on the national legislation on tax fraud, corruption, money laundering and criminal compliance with reference to cybercrime. Wolters Kluwer Hungary, 2019, 288-303.

12 Siehe zur Änderung des Kreises der Geldwäsche-Vortaten in Ungarn: UDVARHELYI Bence - JACSÓ Judit: A pénzmosás alapcselekményi körének változása különös tekintettel az adócsalás problematikájára. Miskolci Doktoranduszok Jogtudományi Tanulmányai, Band 14, Miskolc, 2014, 348-368.

13 Moneyval ist der Expertenausschuss des Europarates zur Bewertung von Maßnahmen gegen Geldwäsche und Terrorismusfinanzierung. 


\section{BEGRIFF UND WURZEL DER (CRIMINAL) COMPLIANCE}

Der Begriff der Compliance hat seine Wurzel ebenfalls in den USA; im Vergleich zur Geldwäsche ist sie jedoch etwas später in den Mittelpunkt des Interesses der Forschung getreten. Die Geldwäsche und die Compliance stehen in engem Zusammenhang. In diesem Teil der Arbeit werden diese Aspekte erläutert.

Compliance leitet sich von dem Verb ,to comply with “ (einhalten) ab, bedeutet ein Verhalten, das mit bestehenden Regelungen übereinstimmt. ${ }^{14}$ Das „Mutterland“ der Compliance ist die USA. ${ }^{15}$ Wie bei der Geldwäschebekämpfung haben die USA auch bei der Compliance eine Vorreiterrolle. In seiner Entwicklungsgeschichte ist das US-amerikanische Gesetz, der sog. „,Foreign Corrupt Practices Act“ (FCPA) aus dem Jahre 1977, von Bedeutung. Der FCPA verbietet im Allgemeinen die Zahlung von Schmiergeldern an ausländische Beamte, um Geschäfte zu erwerben oder zu behalten, und hat zudem eine Reihe von Buchführungspflichten eingeführt. Ihre Bedeutung liegt darin, dass im Ausland begangene Korruptionshandlungen durch Beamte amerikanischer Unternehmen, die auf internationalen Märkten vertreten sind, kriminalisiert wurden. ${ }^{16}$ Die Bedeutung des FCPA wird durch seine extraterritoriale Geltung verstärkt. Zu erwähnen sind noch die U.S. Federal Sentencing Guidelines $\left(U S S G^{17}\right)$. Die Etablierung eines effektiven Compliance und Ethik Programmes ist seit 1991 gemäß den USSG eine wichtige Voraussetzung, um in Strafprozessen gegen eine Organisation eine Strafmilderung zu erreichen. ${ }^{18}$ Zusammengefasst ist zu sagen, dass sich als rechtlich-betriebswirtschaftlicher Begriff die Compliance seit den 90-er Jahren entwickelte und auch in Europa übernommen wurde. ${ }^{19}$

Sucht man nach der kurzen Beschreibung des Begriffes, findet man Folgendes: Der Begriff der Compliance ist nach dem Duden Wörterbuch ,,regelgerechtes, vorschriftsgemäßes, ethisch korrektes Verhalten “ ${ }^{20}$ Er bedeutet ebenso „Folgebereitschaft", „Einhaltung “ oder „Konformität" “. ${ }^{21}$ Nach dem Gabler Wirtschaftslexikon wird sie als ,Einhaltung von Gesetzen, Regeln und Normen “ beschrieben. ${ }^{22}$ Daraus

14 Recherchiert man bei Google findet man fast eine Milliarde Ergebnisse. Vor 10 Jahren ergab diese Suche noch 115000 000. Siehe: RoTSCH, Thomas: Criminal Compliance. Zeitschrift für Internationale Strafrechtsdogmatik (ZIS), 2010/10, 614.

15 SCHRÖDER, Thomas: Compliance an Universitäten - ein Albtraum oder überfälliges Strukturelement? Zu den möglichen Vor- und Nachteilen organisierter Regelbefolgung an öffentlichen Universitäten aus der Perspektive des deutschen Rechts. Zeitschrift für Internationale Strafrechtsdogmatik (ZIS), 2017/6, 279; MOOSMAYER, Klaus: Compliance. Praxisleitfaden für Unternehmen. Beck Verlag, 2021, 4. Auflage, Beck online Datenbank (abgerufen am 4. 10. 2021).

16 AMBRUS- FARKAS: a.a.O., 22-38.

17 https://www.ussc.gov/sites/default/files/pdf/guidelines-manual/2018/GLMFull.pdf (abgerufen am 14. 3. 2021).

18 SCHRÖDER, Thomas: a.a.O., 279.

19 SCHRÖDER, Thomas: a.a.O., 279.

20 https://www.duden.de/rechtschreibung/Compliance (abgerufen am 7. 6. 2021).

21 SCHRÖDER, Thomas: a.a.O., 279.

22 https://wirtschaftslexikon.gabler.de/definition/compliance-27721 (abgerufen am 7. 6. 2021). 
lässt sich schließen, dass der Begriff der Regeln weit zu verstehen ist: Darunter fallen nicht nur die gesetzlichen Vorschriften, sondern auch z. B. die internen Regelungen der Unternehmen und die Empfehlungen internationaler Organisationen.

Der Begriff war am Anfang auf das Gesundheitswesen ${ }^{23}$ beschränkt. Heutzutage hat er aber einen viel breiteren Anwendungsbereich. Compliance-Programme zur Verhinderung von Rechtsverstößen, werden schon seit längerem diskutiert, insbesondere im Bereich des Umwelts- und Arbeitsschutzes, der Korruption und der Kartellverstößen. ${ }^{24}$ Durch „Compliance-Programme“, „Compliance-Richtlinien“ oder „Compliance-Abteilungen" versuchen die Unternehmen den ethischen und rechtlichen Anforderungen zu entsprechen. Die Etablierung von Compliance-Maßnahmen bei Unternehmen und Organisationen hängt eng damit zusammen, dass in den vergangenen Jahren die rechtlichen Anforderungen infolge der Globalisierung und Internationalisierung stark gestiegen und komplexer geworden sind. Die internen Regelungen müssen diesen rechtlichen Anforderungen entsprechen und noch dazu muss erreicht werden, dass die Mitarbeiter die internen Vorschriften bei ihrer Arbeit auch einhalten.

Compliance wird heutzutage als Beschreibung für das ,,Verhalten in Übereinstimmung mit" verwendet, das Einhalten von bestimmten Regeln, welche sowohl rechtliche Normen als auch andere „regulative“ Vorgaben, Standards, Richtlinien und Empfehlungen bedeuten können. ${ }^{25}$ In manchen Fällen können die letztgenannten aus gesetzlichen Vorschriften resultieren. Die Vorschriften sind entweder vom Gesetzgeber oder von der Unternehmensleitung vorgegeben, letzteres kann auch der Einhaltung der gesetzlichen Normen dienen. Es ist wichtig zu betonen, dass Compliance entweder auf ,Zwang “ oder auf fakultativem Einhalten beruhen kann. ${ }^{26}$ Es kann aber auch sein, dass beide Formen vorkommen. Unter „Zwang “ ist zu verstehen, dass die Einrichtung von Compliance-Abteilungen oder Einführung von Compliance-Maßnahmen bei bestimmten Branchen gesetzlich vorgeschrieben ist. Ein Beispiel dafür kann die Geldwäsche sein. Es ist noch anzumerken, dass in Ungarn entsprechend der Regierungsverordnung Nr. 39/2019 (XII. 23.) über das interne Kontrollsystem öffentlicher Unternehmen unter bestimmten Voraussetzungen bei den staatlichen Unternehmen als Bestandteil des internen Kontrollsystems eine sog. „Compliance-Unterstützungsorganisationseinheit" eingerichtet werden muss. ${ }^{27}$

23 Darunter ist zu verstehen, dass die Patienten die Anordnungen des Arztes befolgen, sich „, therapietreu “verhalten, um das Ziel der Heilung der Krankheit durch kooperatives Verhalten zu erreichen. Siehe dazu ROTSCH (2010): a.a.O., 614.; GÜNTHER, Thomas: Die Bedeutung von Criminal-Compliance-Maßnahmen für die strafrechtliche und ordnungswidrigkeitenrectliche Ahndung. Duncker \& Humblot, Berlin, 2017, 98.

24 LEITNER, Roman - DANNECKER Gerhard: Geldwäsche-Compliance: Handbuch der Geldwäsche-Compliance für die rechts- und steuerberatenden Berufe. Linde Verlag, Wien, 2009, 39.

25 Petsche Mair: Lexis Nexis. 2. o.

26 Petsche Mair: Lexis Nexis. 2. o.

27 Gemäß § 9 Regierungsverordnung Nr. 339/2019 hat „,der Geschäftsführer eines Unternehmens eine Compliance-Unterstützungsorganisationseinheit bestehend aus Compli- 
Ziel der Compliance ist die Sicherstellung der Einhaltung der rechtlichen Vorschriften und internen Regelungen sowie Verstöße dagegen zu vermieden oder zu verhindern. Durch eine funktionierende Compliance-Struktur im Unternehmen können sich Unternehmen vor Straftaten und vor Verhängung von Bußgeldern schützen. ${ }^{28}$ Compliance wird entweder durch ein Compliance Management System $(C M S)$ oder durch einen „Compliance Officer" durchgeführt. Wichtig ist, zuerst die Compliance-Ziele und Werte festzulegen, an welche sich sowohl die Unternehmensführung als auch die Mitarbeiter halten müssen und demensprechend Compliance-Programme (Maßnahmen) in den Betrieb zu etablieren, durch welche sichergestellt werden kann, dass die gesetzlichen und ethischen Anforderungen im Unternehmen erfüllt werden.

Laut den Autoren István Ambrus und Ádám Farkas basiert die Compliance ,auf verschiedenen gesetzlichen Bestimmungen (Gesetzesnormen) sowie internen, vom Unternehmen selbst aufgestellten - für seine Eigentümer, Mitarbeiter und Vertragspartner - teils auf der Grundlage von Gesetzen und teils auf der Grundlage der Moral und ethischen Gründen (wie gegebenenfalls Verhaltenskodizes) ". ${ }^{29}$ György Kocziszky und Kolos Kardkovács formulieren den Zweck der Compliance als das „Management der Risiken aus Gewährleistung der Regulierung der Transparenz des Unternehmensbetriebs, des sparsamen und effizienten Einsatzes von Ressourcen; Rahmenbedingungen für ethisches unternehmerisches Handeln schaffen, Korruptionsrisiken vorbeugen ". 30

Es soll noch darauf hingewiesen werden, dass in der wirtschaftsstrafrechtlichen Literatur ein neuer Begriff „Criminal Compliance“ oder „kriminalitätsbezogene Compliance " zur Anwendung gekommen ist. ${ }^{31}$ Laut Tobias Günther ist das Kernanliegen von Criminal Compliance „die Einhaltung der Strafgesetze im weiteren

ance-Beratern einzurichten, die ihre Tätigkeit unmittelbar dem Geschäftsführer der Gesellschaft unterstellt. Wenn die Einrichtung einer Compliance-Abteilung die Gesellschaft aufgrund der Art, Größe und der Tätigkeit der Gesellschaft unverhältnismäßig belastet und der Geschäftsführer der Gesellschaft dem Compliance-Inhaber glaubhaft nachweisen kann, dass die Compliance-Aufgaben nicht nachteilig beeinflusst, genügt die Bestellung eines Compliance-Officers. “

Die erste gesetzliche Verpflichtung zur Einrichtung einer unabhängigen Compliance-Organisation erfolgte in Ungarn durch die Änderung des Gesetzes Nr. CCXXXVII von 2013 über die Kreditinstitute und Finanzunternehmen im Jahre 2017. Gemäß § 153/A muss eine sog. ,für die Einhaltung der rechtlichen Vorgaben verantwortliche Organisationseinheit"“ aufgestellt werden (ab 2018).

28 Compliance Definition und Bedeutung für Unternehmen, https://www.haufe.de/compliance/management-praxis/compliance/bedeutung-von-compliance-fuer-unternehmen_230130_474234.html (abgerufen am 12.7.2021).

29 AMBRUS - FARKAS: a.a.O., 19.

30 KOCZISZKY György - KARDKOVÁCs Kolos: A compliance szerepe a közösségi értékek és érdekek védelmében. Akadémiai Kiadó, Budapest, 2020, 20.

31 Siehe ausführlich zum Thema der Criminal Compliance: ROTSCH, Thomas (Hrsg.): Criminal Compliance. Nomos Verlagsgesellschaft, Baden-Baden, 2015. 
Sinne " (d. h. Kern- und Nebenstrafrecht). ${ }^{32}$ Criminal Compliance bedeutet auch eine neue Regulierungsform (,regulierte Selbstregulierung"), damit eine effizientere Steuerungswirkung auf die Wirtschaft und dadurch ein „verbesserter Rechtsgüterschutz in der modernen Gesellschaft" erreicht werden soll. In der einschlägigen deutschen Literatur werden als Ziel der Criminal Compliance aus Perspektive des Unternehmens die „Haftungsvermeidung “ (,Ahndungsvermeidung “) und aus Sicht des Gesetzgebers die Kriminalitätsprävention genannt. ${ }^{33}$

Ein wesentlicher Bestandteil der Criminal Compliance ist die GeldwäscheCompliance. In diesem Bereich wurden seit mehreren Jahren betriebsinterne Verfahren und Maßnahmen zur Gewährleistung der Einhaltung der einschlägigen Geldwäsche-Vorschriften etabliert. Durch die Geldwäsche-Richtlinien der Europäischen Union wurde seit den 90-er Jahren den Mitgliedstaaten auferlegt, präventiv wirkende Verpflichtungen in den nationalen Vorschriften einzuführen. Das risikobasierte Regelungskonzept wurde auf europäischer Ebene erst mit der III. Geldwäsche-Richtlinie ${ }^{34}$ eingeführt. Im Hintergrund steht der Gedanke, dass die Gefahr der Geldwäsche und Terrorismusfinanzierung nicht in allen Fällen gleich hoch ist. Gemäß dem risikobasierten Ansatz wurden in bestimmten Fällen vereinfachte Sorgfaltspflichten (z. B. bei Sammelkonten von Notaren) und in anderen Fällen, in denen ein erhöhtes Risiko besteht (bei politisch exponierten Personen), erhöhte Sorgfaltspflichten gegenüber Kunden eingeführt. Die Erfüllung dieser Pflichten erfordert nicht nur die Kenntnis der Geldwäscherisiken, sondern auch das Wissen darum, welche Pflichten sich in konkreten Situationen ergeben. Das regelrechte Verhalten kann durch Compliance-Programme unterstützt werden und in manchen Bereichen wird dies sogar als Pflicht festgelegt. Die III. Geldwäsche-Richtlinie enthält auch Regelungen über Sanktionen, wonach die Mitgliedstaaten sicherstellen sollen, dass der Richtlinie unterliegende natürliche und juristische Personen für Verstöße gegen die nach dieser Richtlinie erlassenen nationalen Vorschriften verantwortlich gemacht werden können. ${ }^{35}$ Die Maßnahmen und Sanktionen sollen „wirksam, verhältnismäßig und abschreckend“ sein. Im folgenden Kapitel werden die heute geltenden Regelungen bzgl. der Sanktionierung der Verstöße gegen die Geldwäsche-Vorschriften untersucht, wobei eine klare Trennung zwischen verwaltungsrechtlichen und strafrechtlichen Folgen gezogen werden muss.

GÜNTHER: a.a.O., 101.

GÜNTHER, a.a.O., 101-102.

34 Richtlinie 2005/60/EG des Europäischen Parlaments und des Rates vom 26. Oktober 2005 zur Verhinderung der Nutzung des Finanzsystems zum Zwecke der Geldwäsche und der Terrorismusfinanzierung (Text von Bedeutung für den EWR), ABl L 309 v. 25. 11. 2005.

35 Art. 39 Richtlinie 2005/60/EG. 


\section{DIE VERANTWORTUNG VON UNTERNEHMEN FÜR DIE EINHALTUNG VON GELDWÄSCHEVORSCHRIFTEN}

\subsection{Einführung}

Die Geldwäsche stellt heute noch immer ein erhebliches Bedrohungspotenzial für die Stabilität und Integrität des Finanzsystems der Europäischen Union dar. Der Kampf wurde durch die vor 30 Jahren erlassene erste Geldwäsche-Richtlinie ${ }^{36}$ aufgenommen. Als präventives Mittel gegen die Geldwäsche ist die Richtlinie (EU) 2015/849 des Europäischen Parlaments und des Rates zur Verhinderung der Nutzung des Finanzsystems zum Zwecke der Geldwäsche und der Terrorismusfinanzierung ${ }^{37}$ aus dem Jahre 2015 zu erwähnen, welche $2018^{38}$ durch die fünfte Geldwäsche-Richtlinie geändert wurde. Beide Rechtsakte trugen den durch die Digitalisierung und Fortentwicklung der Technik gestellten Anforderungen im Kampf gegen die Geldwäsche Rechnung.

Die vom europäischen Gesetzgeber verwendbaren legislativen Mittel wurden durch den Vertrag von Lissabon erweitert. ${ }^{39}$ Einen wichtigen Meilenstein im Kampf gegen die Geldwäsche stellt die 2018 erlassene Richtlinie über die strafrechtliche Bekämpfung der Geldwäsche dar, die durch die Mitgliedstaaten bis zum 3. Dezember 2020 umzusetzen war. ${ }^{40}$ Dies erfolgte in Deutschland durch das Gesetz zur Verbesserung der strafrechtlichen Bekämpfung der Geldwäsche vom 09. 3. 2021 und in Ungarn durch das Gesetz Nr. XLIII von 2020, von dem die nationalen Strafgesetzbücher betroffen waren. ${ }^{41}$

36 Richtlinie des Rates (91/308/EWG) vom 10. Juni 1991 zur Verhinderung der Nutzung des Finanzsystems zum Zwecke der Geldwäsche, ABl. L 166 v. 28. 6. 1991.

37 Richtlinie (EU) 2015/849 des Europäischen Parlaments und des Rates vom 20. Mai 2015 zur Verhinderung der Nutzung des Finanzsystems zum Zwecke der Geldwäsche und der Terrorismusfinanzierung, zur Änderung der Verordnung (EU) Nr. 648/2012 des Europäischen Parlaments und des Rates und zur Aufhebung der Richtlinie 2005/60/EG des Europäischen Parlaments und des Rates und der Richtlinie 2006/70/EG der Kommission (Text von Bedeutung für den EWR), ABl. L 141 v. 5. 6. 2015.

38 Die Änderung erfolgte durch die Richtlinie (EU) 2018/843 des Europäischen Parlaments und des Rates vom 30. Mai 2018 zur Änderung der Richtlinie (EU) 2015/849 zur Verhinderung der Nutzung des Finanzsystems zum Zwecke der Geldwäsche und der Terrorismusfinanzierung und zur Änderung der Richtlinien 2009/138/EG und 2013/36/EU (Text von Bedeutung für den EWR), ABl. L 156 v. 19. 6. $2018,43$.

39 Vertrag über die Arbeitsweise der Europäischen Union (AEUV), ABl. C 115 v. 9. 5. 2008. Es ist anzumerken, dass die Geldwäsche gemäß Art. 83 Abs 1. AEUV eine zur besonders schweren grenzüberschreitenden Kriminalitätsbereiche gehörende Straftat ist.

40 Richtlinie (EU) 2018/1673 des Europäischen Parlaments und des Rates vom 23. Oktober 2018 über die strafrechtliche Bekämpfung der Geldwäsche, ABl. L 284 v. 12. 11. 2018, 22.

41 Gesetz Nr. XLIII von 2020 Gesetz Änderung der Strafprozessordnung und anderer damit zusammenhängender Gesetze. 


\subsection{Unionsrechtliche Rahmen}

Die Richtlinie (EU) 2015/849 zur Verhinderung der Nutzung des Finanzsystems zum Zwecke der Geldwäsche und der Terrorismusfinanzierung wurde durch die Richtlinie (EU) 2018/843 des Europäischen Parlaments und des Rates ${ }^{42}$ vom 30. Mai 2018 geändert. Die 5. EU-Geldwäscherichtlinie ist am 9. Juli 2018 in Kraft getreten. Sie musste bis zum 1. Januar 2020 vom nationalen Gesetzgeber umgesetzt werden. Dadurch wurden die Anforderungen im Bereich der Geldwäscheprävention für Unternehmen deutlich verschärft. In der Präambel der fünften Geldwäsche-Richtlinie wurde betont, dass die Anonymität virtueller Währungen ihren potenziellen Missbrauch für kriminelle Zwecke ermöglicht. „Zur Bekämpfung der Risiken im Zusammenhang mit der Anonymität sollten die nationalen zentralen Meldestellen die Möglichkeit haben, Informationen einzuholen, die es ihnen ermöglichen, der Identität des Eigentümers von virtuellem Geld virtuelle Währungsadressen zuzuordnen. " ${ }^{43}$ Durch die verstärkten Sorgfaltspflichten der 5. EU-Geldwäscherichtlinie steigt der administrative Aufwand für Verpflichtete deutlich an. Für verpflichtete Unternehmen bedeutet dies weitere Anpassungen und die Evaluierung ihrer internen Maßnahmen zur Prävention von Geldwäsche und Terrorismusfinanzierung. ${ }^{44}$

Es stellt sich die Frage, welche Folgen mit der Nichtbefolgung der GeldwäscheVorschriften (non-Compliance-Verhalten) verbunden sein können. Es besteht die Gefahr der rechtlichen Folgen (Strafrechtliche Haftung bei natürlichen Personen, Sanktionen durch die Aufsichtsbehörde bzw. die bußgeldrechtliche Verantwortung [bei natürlichen und juristischen Personen]) sowie darüber hinaus die Schädigung der Reputation (z. B. durch die Bekanntgabe der angewandten Sanktionen) und nicht zuletzt sind auch die finanziellen Risiken zu erwähnen. Es stellt sich zunächst die Frage, welche Sanktionen und Maßnahmen durch den mitgliedstaatlichen Gesetzgeber anhand der europäischen Vorgaben wegen der Verletzung der präventiven Verpflichtungen einzuführen sind.

\subsection{Bußgeldrechtliche Sanktionen}

Es finden sich im Primärrecht der Europäischen Union ausdrückliche Ermächtigungen für den Rat der Union (auf Vorschlag der Kommission und nach Anhörung des Europäischen Parlaments) supranationale Geldbußen einzuführen. ${ }^{45}$ In diesem

42 Richtlinie (EU) 2018/843 des Europäischen Parlaments und des Rates vom 30. Mai 2018 zur Änderung der Richtlinie (EU) 2015/849 zur Verhinderung der Nutzung des Finanzsystems zum Zwecke der Geldwäsche und der Terrorismusfinanzierung und zur Änderung der Richtlinien 2009/138/EG und 2013/36/EU (Text von Bedeutung für den EWR), ABl L 156 v. 19. 6. 2018, 43.

43 Präambel Nr. 9. Richtlinie (EU) 2018/843

44 https://www.validatis.de/kyc-prozess/news-fachwissen/5-eu-geldwaescherichtlinie/\#fazit (3. 7. 2021).

45 Wie z. B. Art. 103 AEUV und Art. 83 EAG, sowie Art. 132 Abs. 3 AEUV, der die Verhängung von Sanktionen durch die Europäische Zentralbank (EZB) ermöglicht. Siehe 
Unterkapitel werden im Sekundärrecht der Europäischen Union (IV. GeldwäscheRichtlinie) geregelte Vorschriften dargestellt.

Die 4. Geldwäsche-Richtlinie enthält detaillierte Regelungen über die Sanktionsvorschriften, die durch den mitgliedstaatlichen Gesetzgeber einzuführen sind und welche beim Verstoß gegen bestimmte Verpflichtungen anzuwenden sind. ${ }^{46}$ In der Präambel der 4. Geldwäsche-Richtlinie wird darauf hingewiesen, dass die von den Mitgliedstaaten bisher eingeführten unterschiedlichen verwaltungsrechtlichen Sanktionen und Maßnahmen gegen den Verstoß der wichtigsten präventiven Geldwäsche-Vorschriften voneinander abweichen, was die Bemühungen des einheitlichen Auftritts gegen die Geldwäsche und Terrorismusfinanzierung gefährdet. Daher bezweckt diese Richtlinie die Harmonisierung der mitgliedstaatlichen Regelungen, zumindest bei schweren, wiederholten oder systematischen Verstößen gegen bestimmte Verpflichtungen. Es stellt sich die Frage, welche Pflichten darin einbezogen wurden. Diese sind folgende:

- Sorgfaltspflicht gegenüber Kunden (Art. 10 bis 24),

- Verdachtsmeldungen (Art. 33, 34 und 35),

- Aufbewahrung von Aufzeichnungen (Art. 40) und

- interne Kontrollen (Art. 45 und 46) ${ }^{47}$

Als allgemeine Voraussetzung wird festgelegt, dass diese Sanktionen (Maßnahmen) wirksam, verhältnismäßig und abschreckend sein müssen. Die Mitgliedstaaten können auch strafrechtliche Sanktionen vorsehen (ein Beispiel dafür ist das ungarische Strafrecht, das die vorsätzliche Versäumung der Meldepflicht bestraft), dessen unbeschadet sind verwaltungsrechtliche Sanktionen und Maßnahmen festzulegen. ${ }^{48}$

Die Sanktionen können auch im Fall von juristischen Personen auferlegten Verpflichtungen gegen die Mitglieder des Leitungsorgans und andere natürliche Personen, die nach nationalem Recht für den Verstoß verantwortlich sind, verhängt werden. Die Mitgliedstaaten stellen sicher, dass die zuständigen Behörden mit allen für die Wahrnehmung ihrer Aufgaben erforderlichen Aufsichts- und Ermittlungsbefugnissen ausgestattet sind.

Die verwaltungsrechtlichen Sanktionen und Maßnahmen sollen mindestens Folgendes umfassen:

„a) die öffentliche Bekanntgabe der natürlichen oder juristischen Person und der Art des Verstoßes;

b) eine Anordnung, nach der die natürliche oder juristische Person ihre Verhaltensweise einzustellen und von einer Wiederholung abzusehen hat;

dazu ausführlich: DANNECKER, Gerhard - DANNECKER, Christoph: Europäische und verfassungsrechtliche Vorgaben für das materielle und formelle Unternehmensstrafrecht. Neue Zeitschrift für Wirtschafts-, Steuer- und Unternehmensstrafrecht (NZWiSt), 2016, 162-177.

46 Sanktionen Art. 58-62 Richtlinie (EU) 2015/849 des Europäischen Parlaments und des Rates vom 20. Mai 2015 zur Verhinderung der Nutzung des Finanzsystems zum Zwecke der Geldwäsche und der Terrorismusfinanzierung.

47 Art. 59. Abs. 1 Richtlinie (EU) 2015/849.

48 Art. 58. Abs. 1-2 Richtlinie (EU) 2015/849. 
c) bei Verpflichteten, die einer Zulassungspflicht unterliegen, Entzug oder Aussetzung der Zulassung;

d) vorübergehendes Verbot für jede für den Verstoß verantwortlich gemachte Person, die Leitungsaufgaben bei einem Verpflichteten wahrnimmt, oder jede andere für den Versto $\beta$ verantwortlich gemachte natürliche Person, bei Verpflichteten Leitungsaufgaben wahrzunehmen;

e) maximale Geldbußen in mindestens zweifacher Höhe der infolge des Verstoßes erzielten Gewinne, soweit sich diese beziffern lassen, oder von mindestens 1000000 EUR." 49

Bei Kreditinstituten und Finanzinstituten können sogar schärfere Sanktionen zur Anwendung kommen (im Falle einer juristischen Person maximale Geldbußen sind 5000000 EUR oder 10\% des jährlichen Gesamtumsatzes, im Falle einer natürlichen Person gilt die maximale Geldbuße von 5000000 EUR). ${ }^{50}$

Es ist ersichtlich, dass der Unionsgesetzgeber die Sanktionsnormen, deren Umsetzung den Mitgliedstaaten auferlegt wurden, sehr detailliert bestimmt hat. Die Höhe der zu verhängenden Geldbußen sind sehr hoch bestimmt. Die Mitgliedstaaten können sogar die zuständigen Behörden ermächtigen, weitere Arten von verwaltungsrechtlichen Sanktionen oder Geldbußen zu verhängen. In der Präambel wird darauf hingewiesen, dass gemäß dieser Richtlinie auferlegte verwaltungsrechtliche Sanktionen und Maßnahmen und gemäß dem nationalen Recht auferlegte strafrechtliche Sanktionen nicht gegen den Grundsatz ne bis in idem verstoßen. ${ }^{51}$

\subsection{Folgen des Nichteinhaltens der Geldwäsche-Vorschriften in Ungarn}

\subsubsection{Repressive Vorschriften im ungarischen StGB: Geldwäsche und die Nicht- einhaltung der Meldepflicht}

In Ungarn wurde die Strafbarkeit wegen Versäumung der Geldwäsche-Meldepflicht parallel mit der Kriminalisierung der Geldwäsche in das uStGB ${ }^{52} 1994$ eingeführt. Bezüglich des Straftatbestandes der Geldwäsche in Ungarn soll hier nur darauf hingewiesen werden, dass der Tatbestand durch das Strafrechtsänderungsgesetz Nr. LII von 2020 konzeptionell neugestaltet wurde, um die Geldwäsche effektiver zu bekämpfen und die VI. Geldwäsche-Richtlinie umzusetzen. Die Straftat der Hehlerei wurde in den Straftatbestand der Geldwäsche integriert und als ein Fall derselben konzipiert. Im Mittelpunkt der Änderung stand die strafrechtspolitische Überlegung, dass die Kriminalität dann wirksamer bekämpft werden kann, wenn die strafrechtlichen Mittel auf den dadurch erlangten Vermögensgewinn abzielen.

\footnotetext{
Art. 59 Abs. 2 Richtlinie (EU) 2015/849.

Art. 59 Abs. 3 Richtlinie (EU) 2015/849.

Präambel Nr. 59 Richtlinie (EU) 2015/849.

52 Gesetz Nr. IV. von 1978 über das Strafgesetzbuch.
} 
Es muss hervorgehoben werden, dass in Ungarn des Versäumnis der gesetzlich vorgeschriebenen Meldepflicht ${ }^{53}$ - als eine Form der Geldwäscherei - im Gesetz Nr. IX von 1994 kriminalisiert wurde. Die Geldwäsche-Richtlinien ermächtigen die Mitgliedstaaten, zur Verhinderung der Geldwäsche und Terrorismusfinanzierung strengere Vorschriften zu erlassen. Seit dem Strafrechtsänderungsgesetz aus dem Jahre 2001 sind die Straftatbestände der Geldwäsche und Versäumung der Meldepflicht in eigenständigen Paragraphen voneinander getrennt geregelt. Wichtig ist darauf hinzuweisen, dass die Strafbarkeit wegen Fahrlässigkeit ebenfalls gegeben war, jedoch mittlerweile aufgehoben wurde. Nach dem heute geltende $\mathrm{uStGB}^{54}$ ist das vorsätzliche Versäumen der Verdachtsmeldungspflicht in Verbindung mit Geldwäsche strafbar: „Wer seiner mit der Vorbeugung und Verhinderung von Geldwäsche und der Terrorismusfinanzierung verbundenen, gesetzlich vorgeschriebenen Meldepflicht nicht nachkommt, ist wegen eines Vergehens mit Freiheitsstrafe bis zu zwei Jahren zu bestrafen. " ${ }^{55}$ Die Strafnorm ist als Blankettnorm formuliert. Die Meldepflicht wird im Gesetz Nr. LIII von 2017 über die Vorbeugung und Verhinderung von Geldwäsche und Terrorismusfinanzierung (Geldwäsche-Gesetz) geregelt. Gemäß § 30 Geldwäsche-Gesetz müssen die Verpflichteten (Leiter, Beschäftigten und unterstützende Familienangehörige des Dienstleisters), wenn Daten, Fakten und Umstände auftreten, die

a) auf Geldwäsche,

b) auf Terrorismusfinanzierung oder

c) auf die Herkunft von Sachen aus einer strafbaren Handlung verweisen, den Geldwäschebeauftragten unverzüglich schriftlich Meldung erstatten.

Es muss betont werden, dass die kriminalstrafrechtliche Verantwortung von juristischen Personen (so auch von Unternehmen) in Ungarn nicht möglich ist. Ihnen gegenüber können keine Strafen, sondern nur Maßnahmen verhängt werden, welche auch das Vorhandensein einer natürlichen Person voraussetzt. ${ }^{56}$

\subsubsection{Maßnahmen und verwaltungsrechtliche Sanktionen im ungarischen Geld- wäschegesetz}

Das heute geltende ungarische Geldwäsche-Gesetz ${ }^{57}$ ermächtigt die Aufsichtsbehörde bei einer Verletzung der Bestimmungen des Gesetzes vielfältige Maßnahmen

53 Die Meldepflicht wurde durch das Gesetz Nr. XXIV von 1994 über die Verhütung und Bekämpfung der Geldwäsche (I. Ungarische Geldwäsche-Gesetz) eingeführt.

54 Gesetz Nr. C von Jahre 2012 über das Strafgesetzbuch (uStGB).

$55 \S 401 \mathrm{uStGB}$

56 Gesetz Nr CIV von 2001 über strafrechtliche Maßnahmen gegen eine juristische Person. Siehe dazu SÁNTHA, Ferenc: Special modes of criminal liablity in Hungarian criminal law: liability of the heads ov business and superiors. In: SPINELLIS - THEODORAKIS - BILLIS - PAPADIMITRAKOPOUlOS (Hrsg.): Europe in Crisis: Crime, Criminal Justice, and the Way Forward: Essays in Honour of Nestor Courakis. Athens, Sakkoulas Publications, 2017, 477-496.

57 Siehe zur Erläuterung des ungarischen Geldwäsche-Gesetz ausführlich: PAPP Zsófia (Hrsg.): Magyarázat a pénzmosás és a terrorizmus finanszírozása megelözéséröl és megakadályozásáról. Wolters Kluwer Hungary, Budapest, 2019. 
gegen den Dienstleister zu ergreifen ${ }^{58}$, nämlich Ermahnung, Einstellung der Rechtsverletzung, Überarbeitung der internen Regelungen, Widerruf oder Aussetzung der Erlaubnisse (Genehmigungen) zur Tätigkeitsausübung bis zur Beseitigung der Rechtsverletzung, Löschen aus dem Register oder Verbot der Ausübung der Tätigkeit für wenigstens einen Monat und höchstens zwölf Monate. Es ist auch möglich, dass ein Sachverwalter der Finanzaufsicht ernannt wird oder Fortbildungsmaßnahmen vorgeschrieben werden.

Wichtig ist zu betonen, dass durch die Aufsichtsbehörde die Feststellung der Verantwortung des Leiters oder des für eine Rechtsverletzung verantwortlichen Beschäftigten bzw. unterstützenden Familienangehörigen des Dienstleisters angeregt werden kann und dass sogar die Aussetzung der Leiterbefugnis des Leiters des Dienstleisters bis zur Beseitigung der Rechtsverletzung oder der Widerruf der Leiterbefugnis möglich geworden sind.

Neben diesen Maßnahmen kann auch eigenständig eine Geldbuße verhängt werden. Bei Finanzinstituten kann diese zwischen 400000 Forint und bis zu 10 Prozent der jährlichen Nettoumsätze (höchstens von 2 Milliarden Forint) liegen, bei anderen Dienstleistern (z. B. Wirtschaftsprüfer) liegt diese Summe zwischen 100000 Forint und 400 Millionen Forint.

Die Aufsichtsbehörden berücksichtigen bei der Anwendung der Maßnahmen:

a) die Schwere der Rechtsverletzung,

b) das vorsätzliche oder fahrlässige Verhalten der für die Rechtsverletzung verantwortlichen Personen,

c) den Marktanteil des Unternehmens der rechtsverletzenden Person, wenn dieser hinsichtlich der gegebenen Kategorie des Dienstleisters eingeschätzt werden kann,

d) die auf den Dienstleister oder seine Kunden ausgeübte Wirkung der Rechtsverletzung,

e) die von den verantwortlichen Personen der Aufsicht ausübenden Behörde gegenüber bewiesene Kooperation,

f) die Dauer, Wiederholung oder Häufigkeit der Rechtsverletzung. ${ }^{59}$

Die Maßnahme muss auch dann angewendet werden, wenn der Dienstleister eine juristische Person oder Organisation ohne Rechtspersönlichkeit ist und der Beschäftigte oder unterstützende Familienangehörige des Dienstleisters die Bestimmungen dieses Gesetzes so zum Vorteil des Dienstleisters verletzt, dass die Rechtsverletzung durch die Erfüllung der Aufsichts- oder Kontrollpflicht des Leiters des Dienstleisters hätte verhindert werden können. ${ }^{60}$

Entsprechend den Anforderungen der IV. Geldwäsche-Richtlinie muss der Beschluss der Aufsichtsbehörde nach der Übermittlung an den Dienstleister unverzüglich auf ihrer Homepage veröffentlicht werden, damit dadurch wenigstens die Daten

$58 § 69$ Geldwäsche-Gesetz.

$59 \S 69$ Abs. 4 Geldwäsche-Gesetz.

$60 § 69$ Abs. 6 Geldwäsche-Gesetz. 
und Informationen zum Charakter der Verletzung der Vorschrift oder der Mängel bzw. zur rechtsverletzenden Person eingesehen werden können. ${ }^{61}$

Zusammenfassend kann behauptet werden, dass das ungarische Geldwäsche-Gesetz die Anforderungen der Geldwäsche-Richtlinie nicht nur erfüllt, sondern sogar noch weiter geht, da bei den Verstößen gegen Verpflichtungen kein Unterschied bezüglich der Sanktionen und Maßnahmen gemacht wird. Bei den Geldbußen ist sogar eine Mindesthöhe festgelegt, die Geldwäsche-Richtlinie bestimmt hingegen nur die Höchstgrenze der Geldbuße.

\section{ZUSAMMENFASSUNG UND AUSBLICK}

Die Geldwäsche ist nach wie vor ein bedeutendes Problem auf nationaler, europäischer und globaler Ebene. Bei seiner Bekämpfung kommt den Compliance-Maßnahmen eine besondere Bedeutung zu. Zur Einhaltung der geldwäschebezogenen Verpflichtungen können Geldwäsche-Compliance-Maßnahmen wesentlich beitragen. Es ist keine Legaldefinition für den Begriff „Compliance“ zu finden, weder in den nationalen rechtlichen Vorschriften noch in den nationalen Übereinkommen. Dies kannt dadurch erklärt werden, dass der Begriff Compliance nicht auf die rechtlichen Vorgaben beschränkt ist, sondern auch die zu erfüllenden ethischen Anforderungen erfasst. Auch in wirtschaftlicher Hinsicht hat Compliance einen weiter gefassten Inhalt, denn es bedeutet nicht nur die Einhaltung von Gesetzen, sondern auch die Einhaltung der Kriterien der Nachhaltigkeit und Wirtschaftsethik sowie anderer Erwartungen.

Als „Best Practice“ kann aus Deutschland erwähnt werden, dass der Bundesgerichtshof im Mai 2017 entschieden hat, dass ,für die Bemessung der Geldbuße zudem von Bedeutung ist, inwieweit die Nebenbeteiligte ihrer Pflicht, Rechtsverletzungen aus der Sphäre des Unternehmens zu unterbinden, genügt und ein effizientes Compliance-Management installiert hat, das auf die Vermeidung von Rechtsverstößen ausgelegt sein muss ". ${ }^{62}$ Darüber hinaus kann es auch eine Rolle spielen, ob das Unternehmen nach der Entdeckung des Normverstoßes entsprechende Regelungen optimiert und seine betriebsinternen Abläufe so gestaltet hat, dass vergleichbare Normverletzungen zukünftig jedenfalls deutlich erschwert werden. ${ }^{63}$ Es muss aber betont werden, dass allein durch die Integrierung einer Compliance-Organisation die Haftung weder vermieden noch gemildert werden kann. Compliance muss effektiv zur Normkonformität des Unternehmens beitragen. Die strafrechtliche und bußgeldrechtliche Verantwortung kann präventive Wirkung entfalten.

61 § 71 Geldwäsche-Gesetz.

62 BGH, Urteil vom 9. 5. 2017 - 1 StR 265/16, S. 60. https://juris.bundesgerichtshof.de/cgibin/rechtsprechung/document.py?Gericht=bgh\&Art=en\&nr=78723\&pos=0\&anz=1 (abgerufen am 8. 8. 2021).

63 BLumHOFF, Laura: Rectsfolgen für Unternehmen (12. Kapitel). In: ADICK, Martin BÜLTE, Jens (Hrsg.): Fiskalstrafrecht. Straftaten gegen staatliche Vermögen, C.F. Müller, Heidelberg, 2019, 333. 
Zum Schluss soll noch darauf hingewiesen werden, dass die Europäische Kommission ein neues Paket von Legislativvorschlägen am 20. Juli 2021 vorgelegt hat, um die unionsrechtlichen Vorschriften zur Bekämpfung der Geldwäsche (und Terrorismusfinanzierung) zu verstärken und die Aufdeckung verdächtiger Transaktionen und Aktivitäten zu verbessern. Dieses enthält unter anderen einen Vorschlag zur Schaffung einer neuen EU-Behörde zur Bekämpfung der Geldwäsche. ${ }^{64}$ Zudem ist geplant, eine neue Anti-Geldwäsche-Richtlinie zu erlassen, die die IV. GeldwäscheRichtlinie ersetzen wird. ${ }^{65}$ Es ist ersichtlich, dass die Bekämpfung der Geldwäsche weiterhin ein politisches Anliegen mit höchster Priorität ist. Abzuwarten ist, inwieweit die neuesten legislativen Fortschritte zur Effektivität der Bekämpfung der Geldwäsche auf der Ebene der EU beizutragen vermögen.

\section{LITERATURVERZEICHNIS}

[1] AMBRUS István - FARKAS Ádám: A compliance alapkérdései. Az etikus vállalati müködés elmélete és gyakorlata. Wolters Kluwer Hungary Kft., Budapest, 2019.

[2] ARZT, Gunther: Das schweizerische Geldwäscheverbot im Lichte der amerikanischeren Erfahrungen. Schweizerische Zeitschrift für Strafrecht (ZStrR), 1996, 89-109.

[3] BLUMHOFF, Laura: Rechtsfolgen für Unternehmen. In: ADICK, Martin - BÜLTE, Jens (Hrsg.): Fiskalstrafrecht. Straftaten gegen staatliche Vermögen, C.F. Müller, Heidelberg, 2019.

[4] BÜLTE, Jens: Reform des § 261 StGB: Vermeintlich effektive Abschöpfung statt Rechtsstaatlichkeit. Geldwäsche \& Recht, 2021/1, 8-11.

[5] DANNECKER, Gerhard - DANNECKER, Christoph: Europäische und verfassungsrechtliche Vorgaben für das materielle und formelle Unternehmensstrafrecht. Neue Zeitschrift für Wirtschafts-, Steuer- und Unternehmensstrafrecht (NZWiSt), 2016, 162-177.

[6] GÜNTHER, Thomas: Die Bedeutung von Criminal-Compliance-Maßnahmen für die strafrechtliche und ordnungswidrigkeitenrechtliche Ahndung. Duncker \& Humblot, Berlin, 2017.

[7] GURULÉ, Jimmy: The money laundering contor act of 1986. Creating a new federal offence or merely affording federal prosecutor an alternative means of

64 Das Paket bedeutet die Umsetzung des am 7. Mai 2020 vorgelegten Aktionsplans über den verstärkten Kampf gegen die Geldwäsche und Terrorismusfinanzierung.

65 Vorschlag für eine Richtlinie des Europäischen Parlaments und des Rates über die von den Mitgliedstaaten einzurichtenden Mechanismen zur Verhinderung der Nutzung des Finanzsystems zum Zwecke der Geldwäsche und der Terrorismusfinanzierung und zur Aufhebung der Richtlinie (EU) 2015/84. Brüssel, den 20. 7. 2021 COM(2021) 423 final 2021/0250 (COD). 
punishing specified unlawful activity? American Criminal Law Review, Vol. 32/1994-1995, 823-854. https://scholarship.law.nd.edu/cgi/viewcontent.cgi? article $=1014 \&$ context=law_faculty_scholarship (abgerufen am 16. 06. 2021).

[8] JACSÓ, Judit: Bekämpfung der Geldwäscherei in Europa, unter besonderer Berücksichtigung des Geldwäschestrafrechts von Österreich, der Schweiz und Ungarn (Schriftenreihe Sanktionenrecht in Europa, 5. Band, Hrsg.: DANNECKER, G. - HÖPFEL, F. - SCHWARZENEGGER, Ch.). Neuer Wissenschaftlicher Verlag, Berlin-Wien-Zürich, 2007.

[9] JACSÓ Judit: A compliance fogalmáról és szerepéről a gazdasági életben. Miskolci Jogi Szemle, 2019/1, Sonderausgabe, 82-91.

[10] JACSÓ, Judit: Freiheit und Sicherheit im Spiegel der Geldwäschebekämpfung in Europa. In: KARSAI K. - NAGY F. - SZOMORA Zs. (Hrsg.): Freiheit - Sicherheit - (Straf)Recht Beiträge eines Humboldt-Kollegs. V\&R Unipress, Universitätsverlag Osnabrück, 2011, 103-126.

[11] JACSÓ, Judit - UDVARHELYI, Bence: The fight against money laundering in Hungary. In: FARKAS Ákos - DANNECKER, Gerhard - JACSÓ Judit (Hrsg.): Criminal law aspects of the protection of the financial interests of the EU: with particular emphasis on the national legislation on tax fraud, corruption, money laundering and criminal compliance with reference to cybercrime. Wolters Kluwer Hungary, 2019, 288-303.

[12] KOCZISZKY György - KARDKOVÁCS Kolos: A compliance szerepe a közösségi értékek és érdekek védelmében. Akadémiai Kiadó, Budapest, 2020.

[13] LEITNER, Roman - DANNECKER Gerhard: Geldwäsche-Compliance: Handbuch der Geldwäsche-Compliance für die rechts- und steuerberatenden Berufe. Linde Verlag, Wien, 2009.

[14] MOLNÁR Erzsébet: Compliance és az üzleti titok. Miskolci Jogi Szemle, 2021/1, Band 2, 48-63.

[15] Moosmayer, Klaus: Compliance. Praxisleitfaden für Unternehmen. Beck Verlag, 2021, 4. Auflage, Beck online Datenbank (abgerufen am 4. 10. 2021).

[16] PAPP Zsófia (Hrsg.): Magyarázat a pénzmosás és a terrorizmus finanszírozása megelőzéséről és megakadályozásáról. Wolters Kluwer Hungary, Budapest, 2019.

[17] Rotsch, Thomas (Hrsg.): Criminal Compliance. Nomos Verlagsgesellschaft, Baden-Baden, 2015.

[18] RoTSCH, Thomas: Criminal Compliance. Zeitschrift für Internationale Strafrechtsdogmatik (ZIS), 2010/10.

[19] SÁNTHA, Ferenc: Special modes of criminal liability in Hungarian criminal law: liability of the heads ov business and superiors. In: SPINELLIS - THEODORAKIS - BILLIS - PAPADIMITRAKOPOULOs (Hrsg.): Europe in Crisis: 
Crime, Criminal Justice, and the Way Forward: Essays in Honour of Nestor Courakis. Athens, Sakkoulas Publications, 2017.

[20] SCHRÖDER, Thomas: Compliance an Universitäten - ein Albtraum oder überfälliges Strukturelement? Zu den möglichen Vor- und Nachteilen organisierter Regelbefolgung an öffentlichen Universitäten aus der Perspektive des deutschen Rechts. Zeitschrift für Internationale Strafrechtsdogmatik (ZIS), 2017/6.

[21] UdVARHELYI Bence - JACSÓ Judit: A pénzmosás alapcselekményi körének változása különös tekintettel az adócsalás problematikájára. Miskolci Doktoranduszok Jogtudományi Tanulmányai, Band 14, Miskolc, 2014. 